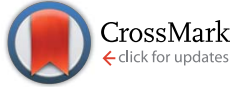

Cite this: RSC Adv., 2017, 7, 16668
Received 13th January 2017 Accepted 5th March 2017

DOI: $10.1039 / \mathrm{c} 7 \mathrm{ra00552k}$

rsc.li/rsc-advances

\section{Nanostructured lipid carrier (NLC)-based novel hydrogels as potential carriers for nepafenac applied after cataract surgery for the treatment of inflammation: design, characterization and in vitro cellular inhibition and uptake studies $\uparrow$}

\begin{abstract}
Shihui Yu, ${ }^{a}$ Guoxin Tan, ${ }^{a}$ Dandan Liu, ${ }^{b}$ Xinggang Yang ${ }^{\star a}$ and Weisan Pan ${ }^{\star a}$
The objective of this study was to design innovative nanostructured lipid carrier (NLC) nanoparticle-loaded hydrogels for drug delivery of nepafenac (NP) applied after cataract surgery for the treatment of postoperative inflammation. NP-NLC nanoparticles were prepared with NP, glycerin monostearate, Miglyol, soy lecithin and Cremophor EL using a melt emulsification method. A two-factor five-level central composite design (CCD) was introduced to perform the experiments. A quadratic function was generated to predict and evaluate the independent variables with respect to the dependent variables. An analysis of variance (ANOVA) statistical test was used to assess the optimization. The morphology of the nanoparticles assessed using TEM revealed a nearly spherical shape. The particle size of the nanoparticles was $92.42 \pm 3.46 \mathrm{~nm}$. DSC analyses indicated that NP was mostly entrapped in the amorphous state. Rheological studies indicated that the gelation temperature of the hydrogels and the NP-NLC hydrogels (NP-NLC-Gel) are $28.5^{\circ} \mathrm{C}$ and $32.0^{\circ} \mathrm{C}$ respectively. At $35^{\circ} \mathrm{C}$ and $\mathrm{pH} 7.4$, the swelling ratio of the NP-NLC-Gel was $8033 \%$. The results of cytotoxicity studies also suggested that NLC-Gel was biocompatible with no significant cytotoxicity observed in the human corneal epithelial cells (HCECs). Preliminary cellular uptake tests proved an enhanced penetration of nepafenac into HCECs when encapsulated in NLCs. The inhibition study suggested that cell uptake was dependant on energy and the clathrin-mediated pathway. Therefore, these results together suggested that the NP-NLC-Gel prepared in this study could be developed as a preliminarily successful carrier for delivering nepafenac for the treatment of inflammation.
\end{abstract}

\section{Introduction}

The eye is a most interesting organ due to its drug disposition characteristics. Generally, topical application of drugs is the method of choice under most circumstances because of its convenience and safety for ophthalmic chemotherapy., ${ }^{\mathbf{1 , 2}}$ However, the bioavailability of topically administered drugs shows significant limitations caused by the rapid and extensive loss of formulation from the precorneal area due to drainage and lacrimal replacement., ${ }^{3,4}$ The strategies to overcome the barriers associated with the targeted ocular delivery of drugs remain a major goal for ocular pharmacotherapy. Considering

\footnotetext{
${ }^{a}$ Department of Pharmaceutics, School of Pharmacy, Shenyang Pharmaceutical University, 103 Wenhua Road, Shenyang 110016, China. E-mail: pppwwwsss@163. com

${ }^{b}$ School of Biomedical \& Chemical Engineering, Liaoning Institute of Science and Technology, Benxi 117004, PR China

$\dagger$ Electronic supplementary information (ESI) available: Experimental details and a few of results data. See DOI: 10.1039/c7ra00552k
}

the poor permeability and transient pre-corneal retention of drugs, increasing transcorneal permeation and prolonging the contact time of formulation have been widely applied in the development of novel ophthalmic dosage forms.

During the last decades, several drug delivery systems, such as liposomes, nanoemulsions, microemulsions, nanoparticles and dendrimers have emerged as novel strategies. ${ }^{5-7}$ Nano- $^{-}$ particle technologies, in general, gather several benefits, e.g., solubilization of hydrophobic and poorly soluble drugs, bioavailability and pharmacokinetic properties improvements, and also protecting drugs from physical, chemical, and/or biological degradation. Recently, nanostructured lipid carriers (NLCs) have shown their applicability as drug delivery systems for ophthalmic therapies. NLCs, as a new generation of lipid nanoparticles, are composed of a solid lipid matrix with a certain amount of a spatially incompatible liquid lipid producing more crystal imperfections in the structure for drug accommodation. ${ }^{8}$ They have many advantages such as controlled drug release, higher drug loading and good bioavailability. Meanwhile, an NLC could promote the pre- 
corneal residence time and corneal absorption through the ocular tissues of the ocular drugs due to their nanoscale dimensions providing them mucoadhesive properties. ${ }^{9-11}$ The main disadvantage of traditional colloidal dispersion systems is their low viscosity, which can be quickly removed by defense mechanisms of the ocular globe. ${ }^{12-14}$

Hydrogels formed by chemical or physical crosslinking are three dimensional structures made from hydrophilic polymers that can imbibe a considerable amount of water while maintaining their integrity. More recently, sensitive hydrogels, in situ gelling, are one of the successful strategies to increase the retention time on ocular surface. ${ }^{15,16}$ Poloxamer 407, also called Pluronics (PF 127) or Lutrol (LF 127) is commercially available triblock copolymers consisting of polyoxyethylene units (70\%) and polyoxypropylene units (30\%). The polymer is more soluble in cold water and aqueous solution of the polymer shows thermo reversible gelation property. ${ }^{17-20}$ However, they generally have a high critical gelation concentration (CGCs) and poor resilience. ${ }^{21,22}$ Carboxymethyl chitosan (CMC) is an attractive biocompatible, non-toxic and biodegradable polymer which is a water-soluble chitosan derivative, prepared by the reaction of chitosan with monochloroacetic acid and, in alkaline condition, has been investigated in some applications, such as pharmaceuticals, foods and cosmetics. ${ }^{23,24}$ To improve the response rate, decrease the critical gelation concentration and high mechanical strength of the hydrogels to meet the requirements of specific applications, a novel hydrogel (F127-CMC) involving poloxamer 407 (F127) and carboxymethyl chitosan (CMC) was synthesized via a crosslinking reaction using glutaraldehyde as the crosslinking agent. The characteristics of the novel hydrogel have been reported in our previous studies. ${ }^{25}$

A cataract is the opacification of the eye lens resulting in a change to the refractive index. Cataract reduces quality of vision by causing blur, glare, monocular diplopia, altered color perception and contrast sensitivity. Cataracts are responsible for $40-60 \%$ of blindness cases worldwide and the majority of these are age related..$^{26,27}$ Because of improvements in intraocular lens design and surgical technique, extracapsular cataract extraction with posterior chamber lens implantation has become an extremely safe and successful procedure. ${ }^{28-30}$ Topically applied non-steroidal anti-inflammatory drugs (NSAIDs) are commonly used in the post- and preoperative antiinflammatory and cystoid macular edema (CME) related to cataract surgery and prevention of intraoperative miosis during cataract surgery. ${ }^{10,31-33}$ Nepafenac (NP) is the only nonsteroidal anti-inflammatory prodrug that requires conversion to its active form, amfenac, through intraocular enzymatic hydrolysis. Amfenac inhibits the activity of both COX-1 and COX-2 enzymes, thus blocking the formation of prostaglandin. Due to this effect, nepafenac was approved by the U.S. FDA to be used in the treatment of pain and inflammation after cataract surgery. After nepafenac penetrates the cornea, bioactive amfenac is formed by means of iris/ciliary object, choroid/ retina. ${ }^{34-36}$

For this purpose, nepafenac-loaded NLC-based dual stimuliresponsive hydrogels (NP-NLC-Gel) were made to develop and evaluate an innovative nepafenac delivery system applied after cataract surgery providing a more corneal absorption and longer contact time. Optimization of NP-NLC formulation was conducted by means of Central Composite Design (CCD). After selecting the critical variables affecting particle size and drug $\mathrm{EE}$, a three-factor five-level central rotatable composite design was employed to plan and perform the experiments. The physicochemical characterization and in vitro release were evaluated, and then the sol-gel transition temperature and swelling behaviour of the hydrogel were assessed. The cytotoxicity and cell uptake of the optimized formulations were also evaluated using the human corneal epithelial cells (HCECs).

\section{Materials and methods}

\subsection{Materials}

Nepafenac (NP) was purchased from Apichem Chemical Tecnology Co., Ltd (Shanghai, China). Glycerin monostearate (GMS) was purchased from Bodi chemical Co., Ltd (Tianjin, China); Miglyol 812N (MIG) and mixtures of Cades, were obtaprylic/capric triglyceriined from Huaxia reagent Co., Ltd (Chengdu, China); Cremophor EL (CRE) was sampled from BASF (Ludwigshafen, Germany). Soy lecithin (SOY) was purchased from Taiwei Pharmaceutical Co., Ltd (Shanghai, China). Ultrafiltration tube (molecular weight cut-off: $3000 \mathrm{Da}$ ) were procured from Xinhua Luyuan Technology Co., Ltd (Beijing, China). Dulbecco's modified Eagle's medium/nutrient mixture F-12 (DMEM/F-12), fetal bovine serum (FBS) and trypsin were all obtained from Gibco Technology (Shanghai, China). Cell Counting Kit-8 (CCK-8) was acquired from Dojindo Molecular Technology Inc. (Shanghai, China). Enhanced BCA Protein Assay Kit and DAPI staining solution were both the products of Beyotime Technology (Jiangsu, China). Nonessential amino acids (NEAA), L-glutamine and penicillinstreptomycin for cell cultures were purchased from Life Technologies. $4 \%$ paraformaldehyde was procured from Solarbio Science \& Technology Co., Ltd (Beijing, China). All other reagents and solvents used were of analytical grade.

\subsection{Preparation of NP-NLC and NP-NLC-Gel}

In the present study, NP-loaded NLC were produced using the melt-emulsification and ultra-sonication techniques., ${ }^{8,9}$ Typically, nepafenac was added to the hot lipid phase consisted of glycerine monostearate (solid lipid) and Miglyol 812N (liquid lipid). Aqueous phase was prepared by dissolving Cremophor EL (surfactant) and soy lecithin (surfactant) in $10 \mathrm{~mL}$ of deionized water. Both phases were heated up to $87^{\circ} \mathrm{C}$ under magnetic stirring. Then the hot surfactant phase was added to the melted oily phase with magnetic stirring at $600 \mathrm{rpm}$ for $15 \mathrm{~min}$ and the primary emulsion was homogenized by probeultrasonic cell disruptor (JY-92-II; Xinzhi, Ningbo, China) for 3 min (active every $3 \mathrm{~s}$ for a $3 \mathrm{~s}$ duration; $400 \mathrm{~W}$ ). Finally, the nanoemulsion was rapidly solidified by placing the container on ice, in order to form NLC. NLC colloidal dispersions were then stored in the refrigerator at $4{ }^{\circ} \mathrm{C}$.

The NP-NLC was loaded into hydrogel using the swellingloading technique..$^{37-39}$ Briefly, dried hydrogels were soaked in 
NP-NLC solution for $24 \mathrm{~h}$ at room temperature and allowed to swell to an equilibrium state in order to achieve a high loading content in the hydrogel. The swollen hydrogels were carefully removed from the drug solution, blotted with filter paper to eliminate surface water, and dried to a constant weight. The dried NP-NLC-Gel was dissolved into the dimethyl sulfoxide and the amount of drug loaded into the hydrogel was determined by high-performance liquid chromatography (HPLC).

The preparation method of NP-Gel was similar to the NPNLC-Gel. Firstly, NP was dissolved into the dimethyl sulfoxide and then used the swelling-loading method. The next steps were same with NP-NLC-Gel.

\subsection{Central composite factorial design (CCD)}

During the preliminary study, the effect of each parameter on the physicochemical properties of the NP-NLC was assessed. After selecting the most important factors, a three factor, five level central composite design was employed to determine the optimum levels of these variables. Three selected independent variables were the nepafenac concentration $\left(\mathrm{NP} \%, X_{1}\right)$, the liquid lipid (Miglyol $812 \mathrm{~N}$ ) concentration (MIG\%, $X_{2}$ ), and the $\mathrm{CRE} / \mathrm{SOY}$ ratio $\left(\mathrm{C} / \mathrm{S}, X_{3}\right)$ respectively. The dependent variables were the mean particle size (PS, $Y_{1}$ ), polydispersity index (PI, $Y_{2}$ ) and entrapment efficiency (EE, $Y_{3}$ ) of the NP-NLC. The upper $(+1)$ and lower $(-1)$ level values of each parameter investigated were offered and values at all levels were given in Table S1. $\dagger$ Formulation factors were evaluated and the resultant NP-NLC properties were optimized in a total of 20 experiments (Table $\mathrm{S} 2 \dagger)$. The CCD method provides a potential advantage in experimental design by adding 6 replicated center points and 6 axial points to the initial factorial design. To analyse the statistical data, the Design-Expert Program 8.2 software was used and analysis of variance (ANOVA) was conducted to determine the significance of the factors and their interactions.

\subsection{Physicochemical characterization}

2.4.1. Mean particle size and polydispersity index. The particle size (PS) and polydispersity index (PI) of NLC were determined by dynamic light scattering (DLS, Zeta-sizer Nano ZS, Malvern Instruments, Malvern, UK) at $25{ }^{\circ} \mathrm{C}$. All samples were diluted with purified water to reach a suitable scattering intensity. Each determination was performed in triplicates.

2.4.2. Determination of encapsulation efficiency. The entrapment efficiency of NP in the NLC was determined indirectly by measuring the concentration of free drug in the aqueous phase of the nanoparticle dispersion. ${ }^{40}$ The drug encapsulation efficiency (EE) was determined using ultrafiltration in centrifuge tubes that were fitted with an ultra-filter (Amicon Ultra, MWCO 3 $\mathrm{kDa}$, Millipore Company). ${ }^{41-43}$ In brief, the NP-NLC $(0.5 \mathrm{~mL})$ was placed in the upper chamber, and the tubes were then centrifuged at $4000 \mathrm{rpm}$ for $30 \mathrm{~min}$. The aqueous phase collected at the sample recovery chamber was subjected to HPLC analysis to determine the NP content. The detection wavelength was $235 \mathrm{~nm}$. The mobile phase consisted of acetonitrile/deionized water/ phosphoric acid (40:60:0.06, v/v/v) and was run at a flow rate

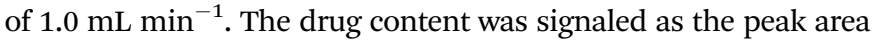

of nepafenac $\left(A_{1}\right)$. Another the same volume of NP-NLC suspension was directly added organic reagent for destroy and the drug content was also determined and signaled as $A_{2}$ following the same procedure. The encapsulation efficiency was calculated as follows:

$$
\mathrm{EE}(\%)=\left(A_{2}-A_{1}\right) / A_{2} \times 100 \%
$$

where EE is the encapsulation efficiency; $A_{1}$ and $A_{2}$ were the peak area of non-entrapped drugs and total drugs in nanoparticle solutions, respectively.

2.4.3. Differential scanning calorimetry (DSC). DSC analysis was performed by a DSC-60 differential scanning calorimeter (Shimadzu, Japan). Appropriate amount of nepafenac, physical mixture and lyophilized NP-NLC were placed in aluminum pans and sealed hermetically. $\mathrm{Al}_{2} \mathrm{O}_{3}$ was used as the reference. The thermal behavior was determined in the range of 30 to $250{ }^{\circ} \mathrm{C}$ at a heating rate of $10^{\circ} \mathrm{C} \mathrm{min}^{-1}$.

2.4.4. Morphological studies. The morphological feature of NP-NLC was observed by transmission electron microscopy (TEM, JEM-1200EX JEOL, Tokyo, Japan). At first, samples were prepared by placing a drop of the NP nanoparticles on a copper grid coated with amorphous carbon film for $2 \mathrm{~min}$ and then negative staining with $1 \%$ phosphotungstic acid. After removing excess solution with filter papers, the grids were dried appropriately and then observed in a transmission electron microscope at $80 \mathrm{kV}$.

\subsection{Measurement of sol-gel transition temperature ( $\left.\boldsymbol{T}_{\text {sol-gel }}\right)$}

To simulate the in vivo sol-gel transition, firstly, the different formulations were mixed with simulated tear fluid (STF) at a ratio of $40: 7$ under moderate stirring at $200 \mathrm{rpm}$ for 15 minutes. ${ }^{12,41}$ The sol-gel transition temperature of NP-NLC-Gel was measured using AR2000ex rheometer (TA Instruments Ltd, USA) in the oscillatory mode. The frequency was fixed at $1 \mathrm{~Hz}$ and samples were subjected to $0.5 \%$ strain using a coneand-plate geometry ( $2^{\circ}$ cone angle, $40 \mathrm{~mm}$ diameter). The samples were heated at a rate of $1{ }^{\circ} \mathrm{C} \mathrm{min}^{-1}$ in a temperature range of $20-40{ }^{\circ} \mathrm{C}$. The measurements were made within the linear viscoelastic region (LVR).

\subsection{Swelling properties}

Hydrogels were dried and weighed $\left(W_{\mathrm{o}}\right)$. The dried hydrogels were immersed in a $5 \mathrm{~mL}$ PBS solution at different temperatures $(25$ or $35{ }^{\circ} \mathrm{C}$ ) and $\mathrm{pH}$ (2.5 or 7.4). At predetermined times, the surface of the swollen hydrogel was wiped dry with a filter paper and its weight $\left(W_{\mathrm{s}}\right)$ was determined. Swelling ratio of hydrogels was then determined as the following ratio: swelling ratio\% $=\left[\left(W_{\mathrm{s}}-W_{\mathrm{o}}\right) /\right.$ $\left.W_{\mathrm{o}}\right] \times 100 . W_{\mathrm{s}}=$ weight of wet sample. $W_{\mathrm{o}}=$ weight of dry sample.

The obtained results represent the average of three comparable experiments for each sample.

\subsection{In vitro release studies}

In vitro release characteristic of different formulations was assessed using a modified dialysis membrane diffusion technique. ${ }^{\mathbf{4} 44}$ The studies were performed with $\mathrm{pH} 7.4$ PBS containing $1 \%(\mathrm{w} / \mathrm{v})$ Tween 80 as release medium. An accurately 
weighed amount of NP eye drops, NP hydrogel, NP-NLC dispersions and NP-NLC hydrogels containing $0.2 \mathrm{mg}$ nepafenac was transferred to a pre-soaked cellulose membrane (8000$14000 M_{\mathrm{wt}}$ ) and immersed in the dissolution flask containing $100 \mathrm{~mL}$ pH 7.4 PBS solutions. The cellulose membranes were fixed to the paddles and rotated at a constant speed (50 rpm). At predetermined times, $1 \mathrm{~mL}$ of release medium was withdrawn and equal volume of fresh medium was added to maintain the constant volume. The amount of released NP was determined by HPLC (Hitachi, Tokyo, Japan) at $235 \mathrm{~nm}$. All release experiments were performed at $34{ }^{\circ} \mathrm{C}$ and repeated three times.

\subsection{Cell cultures}

Human corneal epithelial cells (HCECs) were received from Affiliated Fourth Hospital of China Medical University (Shenyang, China). The cells were cultured in $25 \mathrm{~cm}^{2}$ culture flasks containing DMEM/F-12 medium supplemented with $10 \%$ fetal bovine serum, $1 \%$ L-glutamine, $100 \mathrm{U} \mathrm{mL}^{-1}$ penicillin $\mathrm{G}$ and $100 \mu \mathrm{g} \mathrm{mL}^{-1}$ streptomycin and $1 \%$ NEAA at $37{ }^{\circ} \mathrm{C}$ in a humidified incubator containing $5 \% \mathrm{CO}_{2}-95 \%$ air. The medium was changed every 2 days, and the cells were subcultured after reaching 80-90\% confluence using trypsin $(0.25 \%)$-EDTA $\left(0.5 \mathrm{mmol} \mathrm{L}^{-1}\right)$. HCECs before passages 10 were used in this study.

2.8.1. Cell cytotoxicity assay. Cell viability was measured by the Dojindo's highly water-soluble tetrazolium salt Cell Counting Kit-8 (CCK-8), WST-8 (2-(2-methoxy-4-nitrophenyl)-3-(4-nitrophenyl)-5-(2,4-disulfophenyl)-2 $H$-tetrazolium monosodium salt). ${ }^{45,46}$ HCECs were seeded in 96-well plates at the initial density of $10^{4}$ cells per well. After a $24 \mathrm{~h}$ stabilization of the cells, $100 \mu \mathrm{L}$ fresh medium containing different concentrations of NP, blank NLC, blank NLC-Gel, NP-NLC and NP-NLC-Gel $(0.5,1.0$ and $1.50 \mathrm{mg} \mathrm{mL}^{-1}$ ) was added. The cells were incubated with drugs for 24 and $48 \mathrm{~h}$ respectively. Afterwards, $10 \mu \mathrm{L}$ of CCK-8 reagent was added to each well and incubated for $1.5 \mathrm{~h}$ at $37^{\circ} \mathrm{C}$. The absorbance was then measured by reading the plates at $450 \mathrm{~nm}$ utilizing a microplate reader (ELX-800, BIOTEK). Untreated cells served as a $100 \%$ cell viability control and the media served as background reference. The viability of the treated cell cultures was expressed as a percentage of control untreated cell cultures assumed to be $100 \%$.

\subsubsection{Assessment of cellular uptake}

Quantitative study. To investigate the cell uptake of NP solutions, NP-NLC and NP-NLC-Gel, lipophilic Rhodamine B (RhB), as a fluorescent marker, was encapsulated into the NLC to track the behavior of nepafenac. HCECs $\left(10^{5}\right)$ were seeded in 24-well plates and incubated overnight at $37{ }^{\circ} \mathrm{C}$ with $5 \% \mathrm{CO}_{2}$ in a humidified atmosphere. After the cells reached $80 \%$ confluence, the growth medium was replaced by fresh medium containing RhB-eye drops, RhB-Gel, RhB-NLC and RhB-NLC-Gel $\left(1 \mathrm{mg} \mathrm{mL}{ }^{-1}\right.$ ) and the cells were incubated for $0.5,1$ and $4 \mathrm{~h}$ respectively. At the end of the incubation, the cells were rinsed rapidly three times with $0.5 \mathrm{~mL}$ of ice-cold PBS buffer. The cells were lysed at room temperature with $100 \mu \mathrm{L}$ of lysis solution containing $0.5 \%(\mathrm{v} / \mathrm{v})$ Triton X-100 in $0.2 \mathrm{NNaOH}$. Subsequently, the cell lysate $(75 \mu \mathrm{L})$ from each well was transferred to 96-well black plates and then each well added $100 \mu \mathrm{L}$ ethyl alcohol. The
RhB concentration was determined by fluorescence microplate reader (Varioskan Flash, excitation wavelength $530 \mathrm{~nm}$, emission wavelength $590 \mathrm{~nm}$; Thermo-Scientific, MA, USA). The rest of $25 \mu \mathrm{L}$ cell lysate was used for measuring protein content. Protein content was analyzed by Bradford method with bovine serum albumin as a standard. ${ }^{47}$ The uptake of fluorescence was normalized for the protein content of the cells in each well.

Inhibition study. To evaluate the uptake mechanism of nanoparticles, cell uptake studies were then performed under five different inhibitors. For the first condition, HCECs were preincubated just with culture medium at $4{ }^{\circ} \mathrm{C}$ for $1 \mathrm{~h}$. After the preincubation, old medium was removed and added into the fresh medium containing RhB-eye drops, RhB-Gel, RhB-NLC and RhB-NLC-Gel respectively. Then the cells were continued to incubate for $4 \mathrm{~h}$ at $4{ }^{\circ} \mathrm{C}$. For the other four conditions, HCECs were preincubated with the sodium azide $(10 \mathrm{mM})$, colchicines (40 $\left.\mu \mathrm{g} \mathrm{mL} \mathrm{m}^{-1}\right)$, indomethacin $\left(20 \mu \mathrm{g} \mathrm{mL}^{-1}\right)$ and chlorpromazine $\left(10 \mu \mathrm{g} \mathrm{mL}^{-1}\right)$ for $1 \mathrm{~h}$, respectively. Immediately after the end of preincubation, the solution was removed and replaced with the fresh culture medium containing different fluorescence formulations and inhibitors. Then the cells were continued to incubate for $4 \mathrm{~h}$ at $37{ }^{\circ} \mathrm{C}$. The control was cells incubated at $37^{\circ} \mathrm{C}$ without inhibitors. The results were expressed as the inhibition percentage versus control.

Qualitative study. HCE cells were seeded on a glass-bottom dish in the 24 -well plates till $80 \%$ confluence. The fluorescent solution and NLC dispersed in the cell culture medium were added into the wells. After incubation of $0.5 \mathrm{~h}, 1 \mathrm{~h}$ and $4 \mathrm{~h}$ at $37{ }^{\circ} \mathrm{C}$, the cells were washed three times with PBS followed by fixation with $4 \%$ paraformaldehyde for $25 \mathrm{~min}$. The cells were further washed thrice with PBS and the nuclei were stained with DAPI for $5 \mathrm{~min}$ at room temperature. The fixed cell monolayer was finally washed thrice with PBS and was observed using phase contrast illumination with an Olympus IX71 microscope equipped with Zeiss objectives.

\section{Results and discussion}

\subsection{Formulation building and optimization}

The nepafenac concentration (\%), liquid lipid (Miglyol $812 \mathrm{~N}$ ) concentration (\%) and CRE/SOY ratio were evaluated to optimize the preparation of the NP-NLC. Measured response data for all experimental runs of central composite design (CCD) and their coded level of factors were listed in Table S2. $\dagger$ The best-fit model for the responses was the quadratic model, as follows:

$$
\begin{aligned}
Y=A_{0}+A_{1} X_{1} & +A_{2} X_{2}+A_{3} X_{3}+A_{4} X_{1} X_{2}+A_{5} X_{2} X_{3}+A_{6} X_{1} X_{3} \\
& +A_{7} X_{1}{ }^{2}+A_{8} X_{2}{ }^{2}+A_{9} X_{3}^{2}
\end{aligned}
$$

where $Y$ is the dependent variable, $A_{0}$ is the intercept term, $X_{1}-$ $X_{3}$ are the independent variables, and $A_{1}-A_{9}$ are the regression coefficients of the respective variables.

Model selection for response analysis was performed based on the sequential model sum of squares, lack of fit test and model summary statistics. Details were presented in Table S3. $\dagger$ The prob $>F$ value of $P<0.0001$, high $R$-squared value, low standard deviation and lower predicted residual error sum of 
square (PRESS) value suggest to select quadratic models for all analyzing responses $Y_{1}, Y_{2}$ and $Y_{3}$. ANOVA was performed to evaluate the significance of the variable effects and their interactions. It validates the model obtained (quadratic model, $P<$ 0.05) for all responses of NP-NLC, and also provides significant factors affecting these responses. As shown in Table S4, $\uparrow$ the concentration of drug and liquid lipid, and CRE/SOY ratio were considered significant for particle size $\left(Y_{1}\right)$ and encapsulation efficiency $\left(Y_{3}\right)$, whereas for PI $\left(Y_{2}\right)$, the concentration of drug and CRE/SOY ratio were of significance. The models for response $Y_{1}$, $Y_{2}$ and $Y_{3}$ are as follows:

$$
\begin{aligned}
Y_{1}= & 107.53+10.43 X_{1}+8.03 X_{2}-17.54 X_{3}-5.16 X_{1} X_{2} \\
& -10.04 X_{1} X_{3}-1.52 X_{2} X_{3}+8.64 X_{1}^{2}+5.35 X_{3}^{2} \\
Y_{2}= & 0.31+0.048 X_{1}+0.022 X_{2}-0.056 X_{3}-0.034 X_{1} X_{2} \\
& -0.044 X_{1} X_{3}-0.016 X_{2} X_{3}+0.012 X_{1}^{2} \\
& +0.014 X_{2}^{2}+0.050 X_{3}^{2} \\
Y_{3}= & 85.23-6.59 X_{1}-2.12 X_{2}+3.95 X_{3}+4.22 X_{1} X_{2}+7.83 X_{1} X_{3} \\
& -0.72 X_{2} X_{3}-6.82 X_{1}^{2}+1.97 X_{2}^{2}+0.45 X_{3}^{2}
\end{aligned}
$$

The correlation coefficients $(R)$ were higher than 0.9 and the $p$ values were less than 0.05 , indicating that the goodness of fitting was fine and the significant effects were generated from the factors on the responses, respectively. ${ }^{20,48}$ A positive sign represents a synergistic effect, while a negative sign indicates an antagonistic effect. From eqn (1) and (2), negative coefficients of $X_{3}$ in the model refer to a decreasing trend of particle size and PI when the concentration of Cremophor EL increases. The particle size and PI appeared to increase with an increasing amount of soy lecithin. Similarly, the positive coefficients of $X_{1}$ and $X_{2}$ indicate the increase in particle growth and PI with increasing trend of respective factors. As for EE, negative coefficients of $X_{1}$ in the model refer to that the increase of drug concentration sharply lowers the encapsulation efficiency.

Perturbation graphs (Fig. 1) were plotted to find the factors that most affects the responses. A steep curve indicates the sensitivity of a response to that factor, whereas a relatively flat curve shows that the response is insensitive to the change of the factor. In case of Fig. $1 \mathrm{a}$ and $\mathrm{b}$, factor $\mathrm{C}$ shows a steep curvature, while factor $\mathrm{A}$ and $\mathrm{C}$ both exhibit a slight slope. This may illustrate that factor $\mathrm{C}$ is the most significant influencing parameters of response. Whereas, as for Fig. 1c, factor A shows a steep curvature comparing to factor B and $\mathrm{C}$. These results are consistent with the conclusion draw from the ANOVA. The combined effect of factor $X_{1}$, factor $X_{2}$ and factor $X_{3}$ can be further interpreted with the help of 3D response surface plots showing the effect of factors on responses (Fig. 2). As observed in Fig. 2(a-c), the particle size increases with increasing concentration of nepafenac (A) and Miglyol 812N (B), yet decrease with increasing the CRE/SOY ratio (C). With respect to the sample homogeneity, as shown in Fig. 2(d-f), factors have a similar effect to the PI. However, as for entrapment efficiency, Fig. $2(\mathrm{~g}-\mathrm{i})$ shows that factor $\mathrm{A}$ has the steep curvature, indicating that the concentration of drug is the most significant factor of EE. This can serve as the evidence of the fact that excess drug lead to particle aggregation and increase the PS and PI.

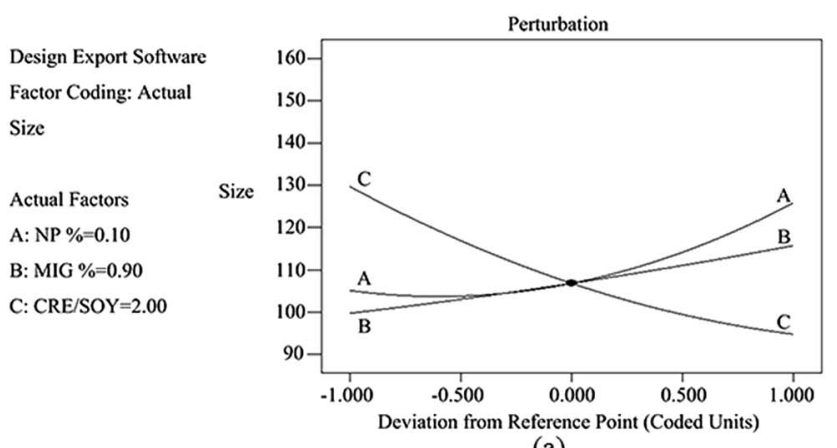

(a)

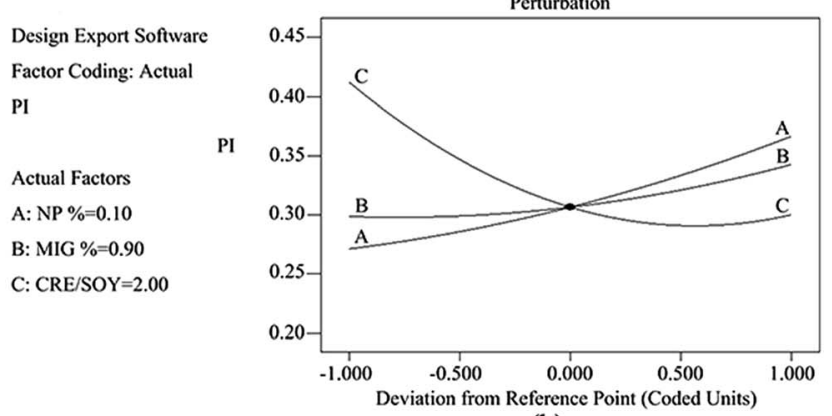

(b)

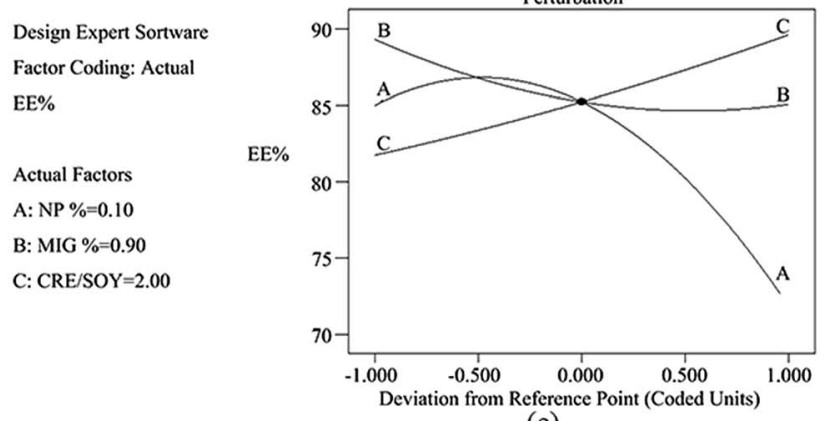

(c)

Fig. 1 Perturbation plots showing effects of $X_{1}(\mathrm{~A}), X_{2}(\mathrm{~B})$ and $X_{3}(\mathrm{C})$ on responses $Y_{1}(\mathrm{a}), Y_{2}$ (b) and $Y_{3}$ (c). $X_{1}$ is factor of nepafenac concentration (NP\%), $X_{2}$ is factor of liquid lipid concentration (MIG\%) and $X_{3}$ is factor of surfactant ratio (CRE/SOY). $Y_{1}$ is response of the mean particle size (PS), $Y_{2}$ is response of polydispersity index $(\mathrm{PI})$ and $Y_{3}$ is response of entrapment efficiency (EE).

The central composite design technique is a very useful tool that provides statistical models, which help researchers to understand the interactions between the parameters that have been optimized and also helps them to optimize the effective parameters using the minimum number of experiments. ${ }^{12,49}$ In the optimal formulation, the concentration of nepafenac, concentration of liquid lipid and CRE/SOY ratio were $0.1 \%, 0.7 \%$ and 1.65 , respectively.

\subsection{Morphological studies}

The morphology of the prepared NP-NLCs was observed using transmission electron microscopy. As shown in Fig. 3a, most of the particles were spheroidal and uniform in size. The particle size (PS) was measured using a Zeta-sizer Nano (Malvern 


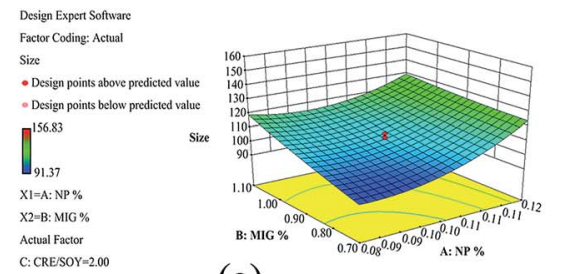

(a)

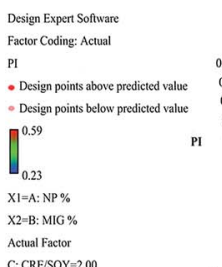

Actual Factor
C: CRESOY $=2.00$

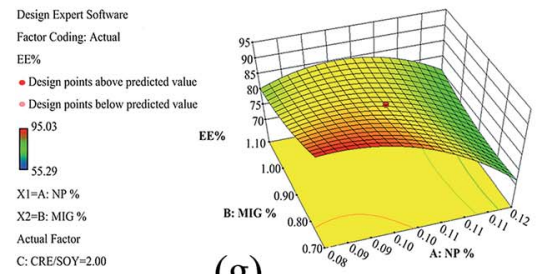

(g) (d)

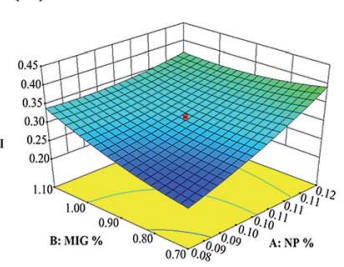

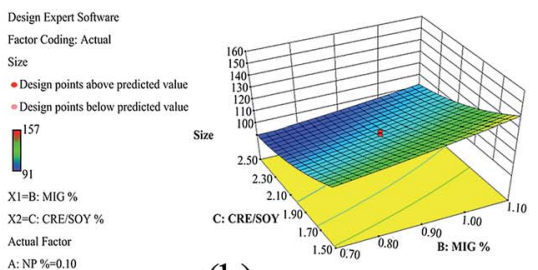

(b)

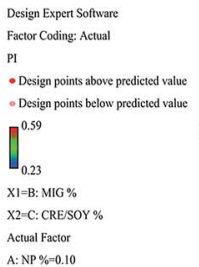

Actual Factor

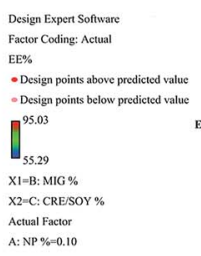

Actual Factor

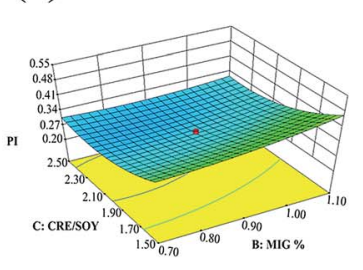

(e)

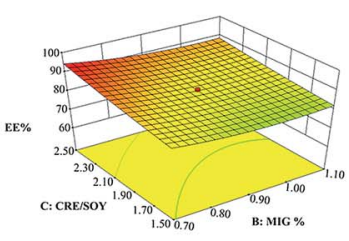

(h)

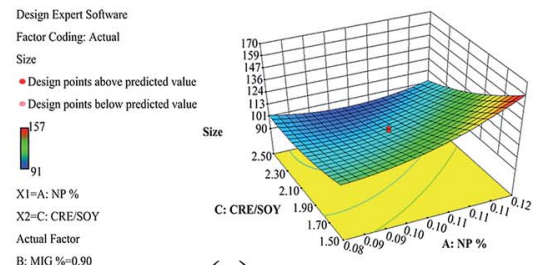

(c)

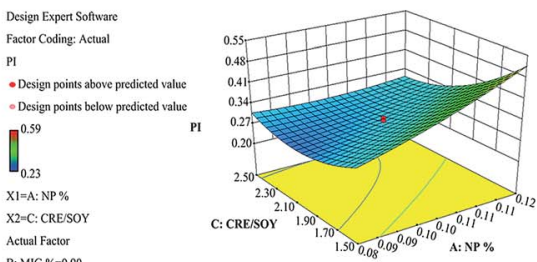

(f)

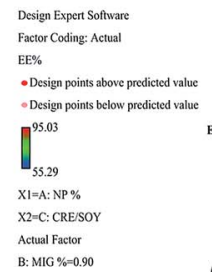

Actual Factor
B. MIC $\%=0.90$

(i)

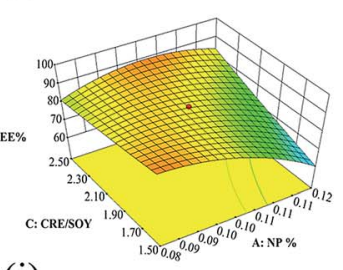

Fig. 2 Three-dimensional (3D) response surface plots showing effects of $X_{1}(\mathrm{~A}), X_{2}(\mathrm{~B})$ and $X_{3}(\mathrm{C})$ on responses $Y_{1}(\mathrm{a}-\mathrm{c}), Y_{2}(\mathrm{~d}-\mathrm{f})$ and $Y_{3}(\mathrm{~g}-\mathrm{i})$. $X_{1}$ is factor of nepafenac concentration (NP\%), $X_{2}$ is factor of liquid lipid concentration (MIG\%) and $X_{3}$ is factor of surfactant ratio (CRE/SOY). $Y_{1}$ is response of the mean particle size (PS), $Y_{2}$ is response of polydispersity index (PI) and $Y_{3}$ is response of entrapment efficiency (EE).

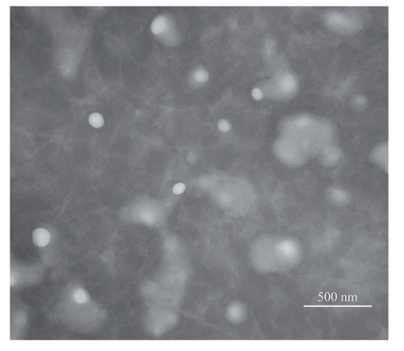

a

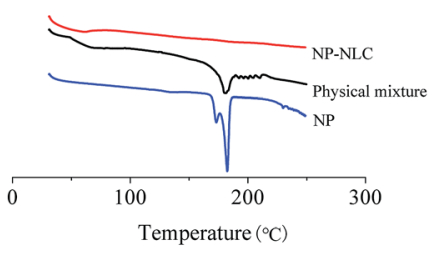

b
Fig. 3 TEM image (a) and DSC thermograms (b) obtained for analysed samples.

Instruments, UK) at $25{ }^{\circ} \mathrm{C}$, which is based on the principle of dynamic light scattering. The particle size was $92.42 \pm 3.46 \mathrm{~nm}$.

\section{3. $\quad$ DSC studies}

Differential scanning calorimetry is an effective method to investigate the melting and crystallization behavior of the lipid molecules and drugs in the nanoparticle preparations. ${ }^{48}$ Fig. $3 \mathrm{~b}$ shows the melting behaviors of nepafenac, physical mixture and lyophilized formulation of NP-NLC. DSC analysis was run heating the sample from $30^{\circ} \mathrm{C}$ to $250^{\circ} \mathrm{C}$. As shown in Fig. $3 \mathrm{~b}$, the endothermic peak of nepafenac was $182{ }^{\circ} \mathrm{C}$. This result emphasizes the chemical stability of NP under the production conditions of drug-loaded NLC. The physical mixture showed a weaker endothermic melting peak at the same position. Nevertheless, the thermogram of NPNLC revealed no melting point, indicating that NP was encapsulated in NLC in a noncrystalline state.

\subsection{Rheological studies}

Phase transition temperature, the temperature at which the liquid phase makes a transition to a gel, is obviously an important parameter for in situ gel-forming systems. ${ }^{50}$ The dynamic properties of the formulation, such as elasticity, or storage modulus $\left(G^{\prime}\right)$, and viscosity, or loss modulus $\left(G^{\prime \prime}\right)$, can provide information about the inherent characteristics of the formulation at room and physiological temperatures. $T_{\text {sol/gel }}$ was chosen as the temperature at which both moduli were equal, reflecting similar elastic and viscous properties. Fig. 4 shows that the gelation temperature of the Gel and the NP-NLCGel are $28.5{ }^{\circ} \mathrm{C}$ (a) and $32.0{ }^{\circ} \mathrm{C}$ (b) respectively. Although the addition of NP-NLC influenced this process, the NP-NLC-Gel had an appropriate gelation temperature. This may be because the mechanism of gelation of poloxamer is based on micelles packing and entanglement, the inclusion of drugs or
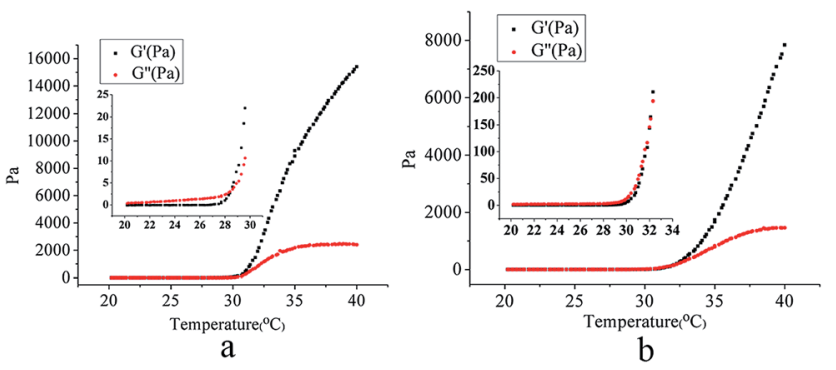

Fig. 4 The gelation temperature of Gel (a) and NP-NLC-Gel (b). 
additives may interfere in micelle formation and, consequently, cause a $T_{\text {sol/gel }}$ modification. ${ }^{\mathbf{5 0 , 5 1}}$

\subsection{Swelling properties}

The swelling is mainly driven by osmotic pressure of hydrophilic and hydrophobic groups inside the polymer network structure. Here pH-dependent swelling is attributable to the fact that polymer contains ionizable moieties (carboxyl groups) along the backbone, and therefore its water absorption capacity is influenced by the acidity or alkalinity of the swelling buffer solution. Similarly, temperature-dependent swelling is observed because the hydrogels contained hydrophilic ethylene oxide (EO) and lipophilic propylene oxide (PO) blocks, and, thus, selfassembled into micelles which could change the state of chains depending on the temperature. The swelling behaviors of the prepared hydrogels by varying swelling conditions were studied as a function of time. The hydrogels were loaded into different phosphate buffer solutions (PBS) including (a) $25^{\circ} \mathrm{C}$, pH 5.8, (b) $25{ }^{\circ} \mathrm{C}, \mathrm{pH} 7.4$, (c) $35{ }^{\circ} \mathrm{C}$, pH 5.8 and (d) $35{ }^{\circ} \mathrm{C}, \mathrm{pH}$ 7.4. The numerical part of the sample code represents the \% weight $(\mathrm{w} / \mathrm{v})$ of F127 and CMC. For example, 5.0F127-2.5CMC represents a hydrogel for which the amount of F127 and CMC was 5.0\% and $2.5 \%$, in deionized water.

Fig. 5a shows the swelling behaviour of the 5.0F127-2.5CMC

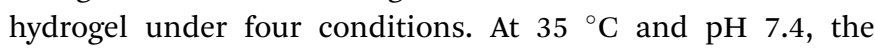
swelling ratio was higher than under the other three conditions. Because at the $\mathrm{pH} 7.4$, the dominant charges in the gels were the unprotonated carboxyl groups which could absorb more water. The poloxamer 407 formed micelles at $35^{\circ} \mathrm{C}$ and this led to gel formation. Under the condition of both $25^{\circ} \mathrm{C}$, pH 5.8 and $35^{\circ} \mathrm{C}$, $\mathrm{pH} 5.8$, the swelling ratios of hydrogels were similar. This indicates that hydrogels are insensitive to temperature under pH 5.8 and it is convenient for transport.

\subsection{In vitro drug release studies}

The release profiles of nepafenac from various formulations are shown in Fig. 5b. As depicted in the Fig. 5b, the NP-NLC-Gel showed a biphasic drug release pattern: a burst release occurred at the initial stage followed by a second slow-release phase. The burst release may be due to desorption and diffusion of the drug from the surface and the difference in structure and melting points between solid lipids and liquid lipids. ${ }^{\mathbf{8} 52}$ The biphasic drug

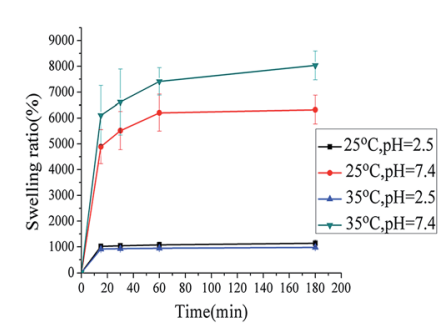

a

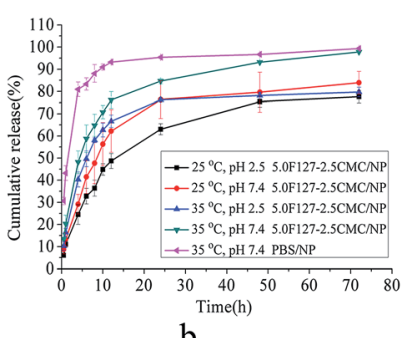

b
Fig. 5 Swelling behaviour of 5.0F127-2.5CMC hydrogel in four conditions (a) and release profiles of NP from four formulations (b). The error bar in the figure indicated the SD $(n=3)$. release is beneficial in terms of anti-inflammatory activity as it helps to achieve the therapeutic concentration of drug in minimal time followed by constant release to maintain sustained release of drug over a prolonged period of time afterwards. We also saw that the release rate of eye drops was slower than the NP-NLC. That may be because the eye drops contain carbomer. In the dry state, carbomer is highly coiled and tightly packed, but once dispersed in water the polymer swells to form a colloidal dispersion that behaves as an anionic electrolyte. Therefore, carbomer has the ability to control the release rate of the drug. ${ }^{53}$ Compared with the release profiles of the eye drops, the developed NLC-Gel can be used as an important platform for sustained drug release.

\subsection{Cytotoxicity and cell uptake studies}

3.7.1. Cytotoxicity studies. The cell viability of HCE cells was tested for each formulation at three concentrations, 0.5, 1.0 and $1.5 \mathrm{mg} \mathrm{mL} \mathrm{m}^{-1}$. Fig. 6(a and b) demonstrated that all nanoparticles formulations showed no toxic effect on cellular viability at all concentrations, up to two days of incubation. However, NP-Gel had significant cytotoxicity at all concentrations compared to untreated cells after incubation for $24 \mathrm{~h}$ and $48 \mathrm{~h}(p<0.05)$. The results were consistent with the corneal hydration levels. That might be explained that NP was dissolved by dimethyl sulfoxide (DMSO) firstly and then loaded into Gel. DMSO is a small amphiphilic molecule which is widely employed in cell biology as an effective penetration enhancer. Therefore, residual DMSO changed the cell membrane permeability and produced cytotoxicity.

\subsubsection{Cell uptake}

Quantitative study. The cell uptake of $\mathrm{RhB}$ hydrogels and nanoparticles was studied in HCE cells as a function of incubation time, as shown in Fig. 6c. The concentration of RhB in four formulations was $1.0 \mathrm{mg} \mathrm{mL} \mathrm{m}^{-1}$. Among four formulations, RhB-eye drops showed the minimum uptake by HCE cells at
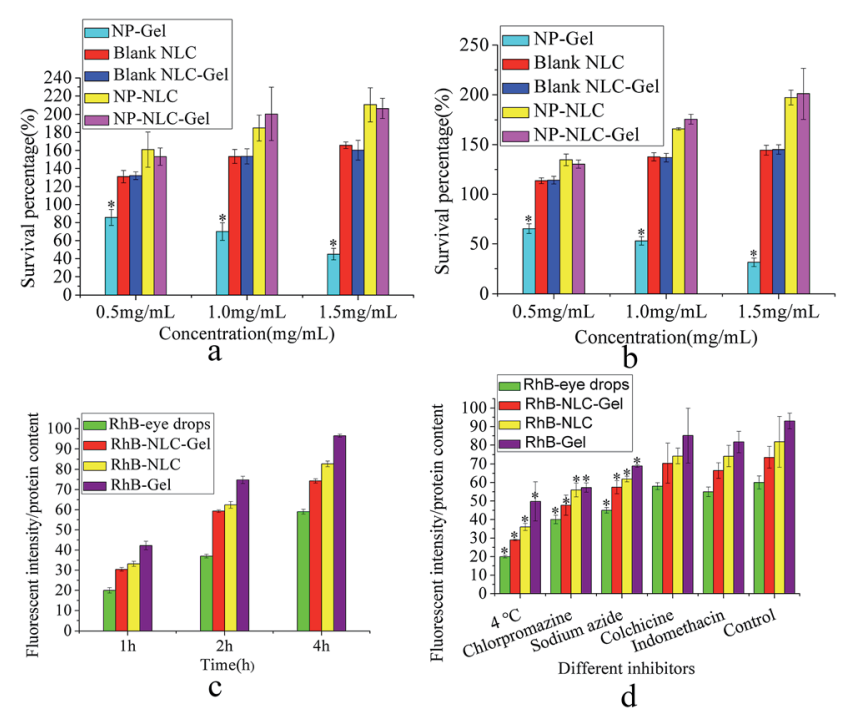

Fig. 6 Cell cytotoxicity of five formulations for $24 \mathrm{~h}$ (a) and 48 h (b), cell uptake of four formulations as a function of incubation time (c) and cell uptake inhibition percentage of four formulations under different blocking conditions for $4 \mathrm{~h}$ (d). 
every time point. The cell uptake of all formulations increased gradually with incubation time. The uptake of RhB-NLC and RhB-NLC-Gel had no significant difference $(p>0.05)$ and this may demonstrate that hydrogels did not affect the cell uptake.

Inhibition study. To further characterize the endocytosis pathways through which the NLC deliver drugs, five endocytosis inhibitors were tested in HCE cells, as shown in Fig. 6d. These inhibitors included chlorpromazine (CPZ, cationic amphipathic molecule that inhibits clathrin disassembly), sodium azide $\left(\mathrm{NaN}_{3}\right.$, a metabolic inhibitor that restrains cellular energy metabolism), colchicine $(\mathrm{CH}$, inhibit endocytosis by interacting with cytoskeleton), $4{ }^{\circ} \mathrm{C}$ (inhibit various transport systems by modulating the membrane's physical state) and indomethacin (caveolaemediated endocytosis inhibitor). ${ }^{47,48,54,55}$ Cell uptake of all formulations was significantly inhibited at $4{ }^{\circ} \mathrm{C}$, with the fluorescent intensity/protein content around $40 \%$. Sodium azide and chlorpromazine also significantly inhibited cell uptake of three formulations, although the inhibition percentage was not as high as those under $4{ }^{\circ} \mathrm{C}$ condition. The remarkable inhibition of cell uptake at $4{ }^{\circ} \mathrm{C}$ was due to the poor membrane fluidity and low metabolic activity, and the cell uptake may be attributed to physical adhesion or binding of nanoparticles to the cell membrane ${ }^{56,57}$ Sodium azide is a metabolic inhibitor that blocks cellular ATP synthesis. The decrease of cell uptake, but not the complete inhibition, could be attributed to the utilization of exogenous ATP by cells and glucose in the media. ${ }^{58}$ The inhibition by $4{ }^{\circ} \mathrm{C}$ condition and sodium azide suggested that the cell uptake was through active endocytosis, an energy-dependent process. Meanwhile, chlorpromazine, using to probe clathrin-mediated endocytosis, showed significant inhibition when compared with control group $(p<0.05)$. Because of the amphipathic nature of the drug, chlorpromazine could incorporate into the lipid bilayer of the plasma membrane increasing lipid fluidity, which in turn may inhibit or block the formation of membrane invaginations, thus leading to a decrease in formulations internalization. ${ }^{58}$ Therefore, chlorpromazine demonstrated the importance of the clathrinmediated pathway for the internalization of formulations. The inhibitors of colchicine and indomethacin showed no significant difference compared with the control group $(p>0.05)$. These

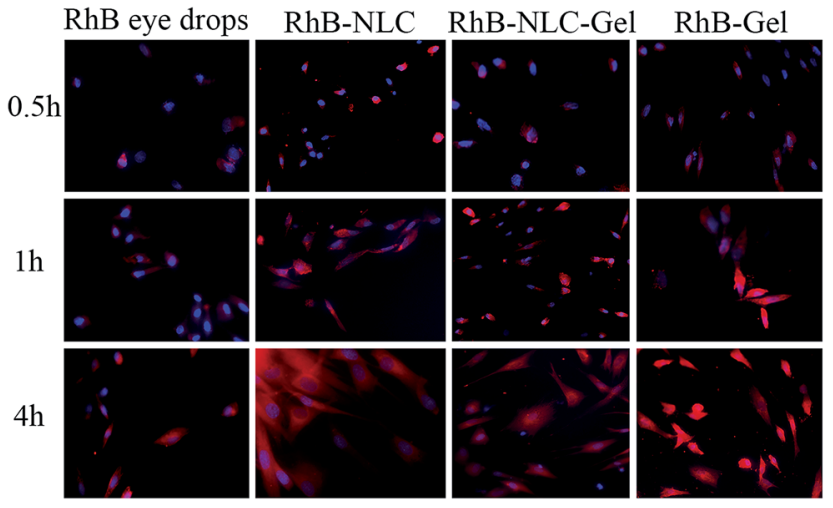

Fig. 7 Fluorescent microscopic images for HCE cells after $0.5 \mathrm{~h}, 1 \mathrm{~h}$ and $4 \mathrm{~h}$ of incubation with RhB eye drops, RhB-NLC, RhB-NLC-Gel and $\mathrm{RhB}-\mathrm{Gel}$. results may demonstrate that caveolae-mediated and micropinocytosis pathways were not the main way for the HCE cells uptaking formulations.

Qualitative study. The qualitative study was carried out using a fluorescent microscope to visualize the cell uptake of different formulations. As shown in Fig. 7, the nanoparticles were clearly internalized into HCE cells, strongly supporting the aforementioned quantitative measurement of cell uptake. The fluorescence intensity of RhB-NLC and RhB-NLC-Gel was similar and the result was consisted with quantitative study.

\section{Conclusions}

We have successfully designed an innovative nanostructured lipid carrier (NLC) nanoparticles-loaded hydrogels for drug delivery of nepafenac (NP) applied after cataract surgery for the treatment of postoperative inflammation. NP-NLC nanoparticles were prepared with NP, glycerine monostearate, Miglyol, soy lecithin and Cremophor EL using melt emulsification with ultrasonic method. A three-factor five-level central composite design (CCD) was introduced to perform the experiments. A quadratic function was generated to predict and evaluate the independent variables with respect to the dependent variables. Analysis of variance (ANOVA) statistical test was used to assess the optimization. In the optimal formulation, the concentration of nepafenac, concentration of liquid lipid and CRE/SOY ratio were $0.1 \%, 0.7 \%$ and 1.65 , respectively. The morphology of nanoparticles assessed by TEM revealed a nearly spherical shape. The particle size was $92.42 \pm 3.46 \mathrm{~nm}$. DSC analyses indicated that NP was mostly entrapped in amorphous state. Rheological studies indicated that the gelation temperature of the hydrogels and the NP-NLC hydrogels (NP-NLC-Gel) are $28.5{ }^{\circ} \mathrm{C}$ and $32.0{ }^{\circ} \mathrm{C}$ respectively. At $35{ }^{\circ} \mathrm{C}$ and $\mathrm{pH} 7.4$, the swelling ratio of NP-NLC-Gel was $8033 \%$. The in vitro drug release demonstrated that NP-NLC sustained the drug release for $24 \mathrm{~h}$ in a biphasic pattern. The results of cytotoxicity suggested that NLC-Gel was biocompatible with no significant cytotoxicity observed in the human corneal epithelial cells (HCECs). Preliminary cellular uptake test proved an enhanced penetration of nepafenac into HCECs when encapsulate in NLC. The inhibition study suggested that the cell uptake was depended on energy and the clathrin-mediated pathway.

On the basis of these results, it can be concluded that this delivery system would provide numerous benefits as compared to those of traditional delivery systems such as eye drops, as it would be more convenient in patients, providing stronger effects for longer time periods. Meanwhile, further studies are required to be done for evaluating feasibility.

\section{Acknowledgements}

The authors have no financial involvement with any organization or entity with a financial interest or conflict with the subject matter and topics discussed in the present review. The authors report no conflict of interest. The authors alone are responsible for the content and writing of the paper. 


\section{Notes and references}

1 A. Gonzalez, L. I. Tartara, S. D. Palma and C. I. Alvarez Igarzabal, Mater. Sci. Eng., C, 2015, 51, 73-79.

2 R. Jeencham, W. Tiyaboonchai and M. Sutheerawattananonda, Asian J. Pharm. Sci., 2016, 11, 136-137.

3 A. A. Abdelrahman, H. F. Salem, R. A. Khallaf and A. M. A. Ali, J. Pharm. Innov., 2015, 10, 254-268.

4 Y. Duan, X. Cai, H. Du and G. Zhai, Colloids Surf., B, 2015, 128, 322-330.

5 R. Agarwal, I. Iezhitsa, P. Agarwal, N. A. Abdul Nasir, N. Razali, R. Alyautdin and N. M. Ismail, Drug Delivery, 2016, 23, 1075-1091.

6 G. Andrei, C. A. Peptu, M. Popa, J. Desbrieres, C. Peptu, F. Gardikiotis, M. Costuleanu, D. Costin, J. C. Dupin, A. Uhart and B. I. Tamba, Int. J. Pharm., 2015, 493, 16-29.

7 R. Liu, S. Wang, S. Fang, J. Wang, J. Chen, X. Huang, X. He and C. Liu, Nanoscale Res. Lett., 2016, 11, 254.

8 W. Zhang, X. Li, T. Ye, F. Chen, X. Sun, J. Kong, X. Yang, W. Pan and S. Li, Int. J. Pharm., 2013, 454, 354-366.

9 H. Almeida, P. Lobão, C. Frigerio, J. Fonseca, R. Silva, P. Quaresma, J. M. S. Lobo and M. H. Amaral, J. Drug Delivery Sci. Technol., 2016, 35, 69-80.

10 E. B. Souto, S. Doktorovova, E. Gonzalez-Mira, M. A. Egea and M. L. Garcia, Curr. Eye Res., 2010, 35, 537-552.

11 N. Ustundag-Okur, E. H. Gokce, D. I. Bozbiyik, S. Egrilmez, O. Ozer and G. Ertan, Eur. J. Pharm. Sci., 2014, 63, 204-215.

12 J. Hao, X. Wang, Y. Bi, Y. Teng, J. Wang, F. Li, Q. Li, J. Zhang, F. Guo and J. Liu, Colloids Surf., B, 2014, 114, 111-120.

13 Q. Luo, J. Zhao, X. Zhang and W. Pan, Int. J. Pharm., 2011, 403, 185-191.

14 A. C. Silva, M. H. Amaral, E. Gonzalez-Mira, D. Santos and D. Ferreira, Colloids Surf., B, 2012, 93, 241-248.

15 P. A. Faccia, F. M. Pardini and J. I. Amalvy, eXPRESS Polym. Lett., 2015, 9, 554-566.

16 S. Kirchhof, A. M. Goepferich and F. P. Brandl, Eur. J. Pharm. Biopharm., 2015, 95, 227-238.

17 D. Achouri, M. Sergent, A. Tonetto, P. Piccerelle, V. Andrieu and V. Hornebecq, Drug Dev. Ind. Pharm., 2015, 41, 493-501.

18 R. Barse, C. Kokare and A. Tagalpallewar, J. Drug Delivery Sci. Technol., 2016, 33, 66-74.

19 M. Dewan, B. Bhowmick, G. Sarkar, D. Rana, M. K. Bain, M. Bhowmik and D. Chattopadhyay, Int. J. Biol. Macromol., 2015, 72, 706-710.

20 W. Huang, N. Zhang, H. Hua, T. Liu, Y. Tang, L. Fu, Y. Yang, X. Ma and Y. Zhao, Biomed. Pharmacother., 2016, 83, 107113.

21 X. J. Loh, S. H. Goh and J. Li, Biomacromolecules, 2007, 8, 585-593.

22 X. Marcos, S. Perez-Casas, J. Llovo, A. Concheiro and C. Alvarez-Lorenzo, Int. J. Pharm., 2016, 500, 11-19.

23 A. L. Bukzem, R. Signini, D. M. Dos Santos, L. M. Liao and D. P. Ascheri, Int. J. Biol. Macromol., 2016, 85, 615-624.

24 V. C. Dumont, A. A. Mansur, S. M. Carvalho, F. G. Medeiros Borsagli, M. M. Pereira and H. S. Mansur, Mater. Sci. Eng., C, 2016, 59, 265-277.
25 S. Yu, X. Zhang, G. Tan, L. Tian, D. Liu, Y. Liu, X. Yang and W. Pan, Carbohydr. Polym., 2017, 155, 208-217.

26 S. Cetinel and C. Montemagno, Asia-Pac. J. Ophthalmol., 2015, 4, 381-387.

27 S. E. Skalicky, K. R. Martin, E. Fenwick, J. G. Crowston, I. Goldberg and P. McCluskey, Clin. Exp. Ophthalmol., 2015, 43, 335-341.

28 H. Hashemi, M. A. Seyedian and M. Mohammadpour, Curr Opin Ophthalmol., 2015, 26, 3-9.

29 S. A. Obstbaum, J. Cataract Refractive Surg., 1992, 18, 219231.

30 R. Schalnus and R. Schalnus, Ophthalmologica, 2003, 217, 89-98.

31 R. S. Hoffman, R. Braga-Mele, K. Donaldson, G. Emerick, B. Henderson, M. Kahook, N. Mamalis, K. M. Miller, T. Realini, N. H. Shorstein, R. K. Stiverson, B. Wirostko, A. C. C. Committee and S. the American Glaucoma, J. Cataract Refractive Surg., 2016, 42, 1368-1379.

32 S. Sethi, D. Chalia, M. Chalia and M. Kaur, Int. J Basic. Clin. Pharmacol., 2015, 4, 98.

33 T. Tsukamoto, K. Hironaka, T. Fujisawa, D. Yamaguchi, K. Tahara, Y. Tozuka and H. Takeuchi, Asian J. Pharm. Sci., 2013, 8, 104-109.

34 F. Afrashi, A. S. Hashas, C. Shahbazov, M. Arici, M. S. Yikilmaz, R. Deveci, S. Karacali and U. Sahar, J. Ocul. Pharmacol. Ther., 2015, 31, 43-50.

35 T. P. O'Brien, Curr. Med. Res. Opin., 2005, 21, 1131-1137.

36 S. Usman, M. Akram, A. Aziz, V. Ramesh and O. A. Sarheed, Pak. J. Pharm. Sci., 2014, 27, 1541-1546.

37 H. W. Huh, L. Zhao and S. Y. Kim, Carbohydr. Polym., 2015, 126, 130-140.

38 Y. Y. Liu and X. D. Fan, Biomaterials, 2005, 26, 6367-6374.

39 D. Silva, L. F. Pinto, D. Bozukova, L. F. Santos, A. P. Serro and

B. Saramago, Colloids Surf., B, 2016, 147, 81-89.

40 C. Vitorino, J. Almeida, L. M. Goncalves, A. J. Almeida, J. J. Sousa and A. A. Pais, J. Controlled Release, 2013, 167, 301-314.

41 A. Hugo, L. Paulo, F. Christian, F. Joel, S. Renata, P.-d.-O. Ana, L. José Manuel Sousa and A. Maria Helena, Curr. Drug Delivery, 2016, 13, 953-970.

42 R. R. Patlolla, M. Chougule, A. R. Patel, T. Jackson, P. N. Tata and M. Singh, J. Controlled Release, 2010, 144, 233-241.

43 R. R. Patlolla and V. Vobalaboina, J. Pharm. Sci., 2005, 94, 437-445.

44 L. Xi, T. Wang, F. Zhao, Q. Zheng, X. Li, J. Luo, J. Liu, D. Quan and J. Ge, PLoS One, 2014, 9, e100632.

$45 \mathrm{H}$. Chen, X. Liang, F. Xu, B. Xu, X. He, B. Huang and M. Yuan, Molecules, 2014, 19, 12048-12064.

46 H.-L. Chen, H.-F. Kao, J.-Y. Wang and G.-T. Wei, Chem. Res. Chin. Univ., 2014, 61, 763-769.

47 T. Sawada, J. Nagai, Y. Okada, R. Yumoto and M. Takano, Eur. J. Pharmacol., 2012, 684, 146-153.

48 T. Guo, Y. Zhang, J. Zhao, C. Zhu and N. Feng, J. Nanobiotechnol., 2015, 13, 47.

49 O. P. Ranjan, U. Y. Nayak, M. S. Reddy, S. J. Dengale, P. B. Musmade and N. Udupa, J. Pharm. Innov., 2014, 9, 95-105. 
50 T. Gratieri, G. M. Gelfuso, E. M. Rocha, V. H. Sarmento, O. de Freitas and R. F. Lopez, Eur. J. Pharm. Biopharm., 2010, 75, 186-193.

51 T. Ur-Rehman, S. Tavelin and G. Grobner, Int. J. Pharm., 2011, 409, 19-29.

52 R. S. Bhatta, H. Chandasana, Y. S. Chhonker, C. Rathi, D. Kumar, K. Mitra and P. K. Shukla, Int. J. Pharm., 2012, 432, 105-112.

53 B. Buchan, G. Kay, A. Heneghan, K. H. Matthews and D. Cairns, Int. J. Pharm., 2010, 392, 192-197.
54 Y. Luo, Z. Teng, T. T. Wang and Q. Wang, J. Agric. Food Chem., 2013, 61, 7621-7629.

55 D. S. Muller, P. Houpert, J. Cambar and M. H. Henge-Napoli, Toxicol. Sci., 2008, 101, 254-262.

56 C. He, Y. Hu, L. Yin, C. Tang and C. Yin, Biomaterials, 2010, 31, 3657-3666.

57 Y. Liu, P. Wang, C. Sun, N. Feng, W. Zhou, Y. Yang, R. Tan, Z. Chen, S. Wu and J. Zhao, Int. J. Pharm., 2010, 397, 155-163. 58 S. E. Gratton, P. A. Ropp, P. D. Pohlhaus, J. C. Luft, V. J. Madden, M. E. Napier and J. M. DeSimone, Proc. Natl. Acad. Sci. U. S. A., 2008, 105, 11613-11618. 